\title{
Generation of a telecom-to-mid-infrared spanning supercontinuum using silicon-on-insulator wire waveguides
}

\author{
Bart Kuyken ${ }^{1}$, Xiaoping Liu' ${ }^{2}$, Richard M. Osgood ${ }^{2}$ Jr., Yurii A. Vlasov ${ }^{3}$, Roel Baets ${ }^{1}$, Gunther Roelkens ${ }^{1}$, \\ and William M. J. Green ${ }^{3}$ \\ ${ }^{1}$ Photonics Research Group, Department of Information Technology, Ghent University - imec, Ghent B-9000, Belgium \\ ${ }^{2}$ Microelectronics Sciences Laboratories, Columbia University, New York, NY 10027, USA. \\ ${ }^{3}$ IBM T. J. Watson Research Center, 1101 Kitchawan Road, Yorktown Heights, NY 10598, USA. \\ Tel.:+32 (0)9 32-9-264-3335.Email: Bart.Kuyken@intec.ugent.be
}

\begin{abstract}
We demonstrate an ultra-broadband optical supercontinuum extending from $1.53 \mu \mathrm{m}$ up to $2.55 \mu \mathrm{m}$, generated in a dispersion-engineered silicon photonic wire. The supercontinuum is generated using low power mid-infrared picosecond pump pulses at $2.12 \mu \mathrm{m}$.

(C) 2011 Optical Society of America

OCIS codes: (130.4310) Nonlinear Waveguides; (320.6629) Supercontinuum Generation
\end{abstract}

\section{Introduction}

Supercontinuum generation, the dramatic spectral broadening of narrow band pulses or continuous wave signals in nonlinear waveguides, has been successfully demonstrated in single mode fibers and photonic crystal fibers [2]. Recently it has also been demonstrated in doped glass [3], chalcogenide [4] and silicon integrated planar waveguides [5]. These continuums were generated at telecommunication wavelengths, primarily in the context of having a broadband source for WDM applications. However, compact broadband integrated sources may also be useful outside telecommunication, e.g. in biomedical applications such as optical coherence tomography and spectroscopy [2]. Silicon-on-insulator integrated waveguide circuits show an enormous potential as a nonlinear platform. The tight optical confinement in silicon wire waveguides, originating from the high refractive index contrast, gives rise to record effective nonlinear parameters [5]. These properties can be fully exploited by working at photon energies near the half band gap of silicon where the nonlinear absorption (two photon absorption) is strongly suppressed, enabling efficient nonlinear devices [6-8]..In this paper a broad supercontinuum is generated from dispersion engineered sub-wavelength silicon photonic wires pumped by pulses at a wavelength of $2.12 \mu \mathrm{m}$. While previous demonstrations of supercontinuum generation from integrated waveguide circuits made use of femtosecond pump pulses [3-5], here the supercontinuum is realized using 2-picosecond-long pump pulses with relatively low power levels $(12.7 \mathrm{~W}$ peak power). The generated supercontinuum extends all the way from the telecom C-band at 1.53 $\mu \mathrm{m}$, up to the mid-infrared at $2.55 \mu \mathrm{m}$.

\section{Experimental results}

The silicon nanophotonic wires used in the experiment were fabricated on a $200 \mathrm{~mm}$ SOI wafer in a CMOS pilot line, consisting of a $220 \mathrm{~nm}$ silicon waveguide layer on a $2 \mu \mathrm{m}$ buried oxide layer. The photonic wire used is $900 \mathrm{~nm}$ wide and has a length of $2 \mathrm{~cm}$ with no top cladding. The high confinement realized in these silicon nanophotonic wires not only results in a high effective nonlinear parameter, but also gives rise to anomalous waveguide dispersion in the mid-infrared. The waveguide dispersion was simulated using a commercial finite element solver (RSoft FemSIM). The waveguide shows low anomalous dispersion $(D>0)$ for the TE polarized mode between the two zero dispersion wavelengths of $1800 \mathrm{~nm}$ and $2410 \mathrm{~nm}$, as shown in Fig. 1(c).

For the experiment, a picosecond pulse train (FWHM $\sim 2 \mathrm{ps,} \mathrm{repetition} \mathrm{rate}=76 \mathrm{MHz}$ ) generated by a tunable optical parametric oscillator (OPO) coupled to a single-mode optical fiber is used as the pump. Coupling into/out of the SOI photonic wire is accomplished using lensed fibers. A polarization controller is used to excite the TE polarized mode. The waveguide loss was found to be $2.5 \mathrm{~dB} / \mathrm{cm}$ for the TE mode at both telecom and mid-infrared wavelengths. The fiber coupling loss at each facet was $9 \mathrm{~dB}$. The output is characterized with a mid-infrared optical spectrum analyzer, at $1 \mathrm{~nm}$ spectral resolution.

Figure 1(a) shows the realized supercontinuum spectrum for different input coupled peak powers. By examining the evolution of the spectrum, particularly at low input power, we can observe that the supercontinuum originates from a number simultaneously occurring nonlinear processes within the silicon wire. For example, at an input power of $3.1 \mathrm{~W}$ two broad sidebands (labeled MI(1)) are generated around the peak wavelengths of $1990 \mathrm{~nm}$ and $2250 \mathrm{~nm}$, immediately adjacent to the pump. Further away from the pump, two additional narrower sidebands are visible (labeled MI(2)), at wavelengths near $1870 \mathrm{~nm}$ and $\sim 2510 \mathrm{~nm}$. The peak at $\sim 2510 \mathrm{~nm}$ is just at the edge of the OSA 
maximum range of $2.5 \mu \mathrm{m}$, but begins to shift to shorter wavelengths for higher input powers. These sidebands labeled MI(1) and MI(2) result from modulation instability and the associated exponential amplification of noise, at wavelengths satisfying the two solutions of the phase matching condition $\beta_{2} \cdot(\Delta \omega)^{2}+(1 / 12) \cdot \beta_{4} \cdot(\Delta \omega)^{4}+2 \gamma P=0$. In this equation, $\beta_{2}$ and $\beta_{4}$ are the second and fourth order waveguide dispersion, $\Delta \omega$ is the detuning of the idler and signal from the pump, $\gamma$ is the nonlinear parameter, and $P$ is the coupled peak pump power. At an input peak power level of $4.3 \mathrm{~W}$, a Raman Stokes peak appears at $2420 \mathrm{~nm}$, red-shifted by approximately $15.6 \mathrm{THz}$ away from the pump. Additionally, the significant broadening of the pump due to self-phase modulation can clearly be seen. At the highest obtainable peak powers of $12.7 \mathrm{~W}$, cascaded four wave mixing products serve to broaden and flatten the spectrum further. The peak appearing at $1700 \mathrm{~nm}$ can be understood as the mixing product between the second order MI point at $1900 \mathrm{~nm}$ and the pump. Finally, the peak appearing at $1600 \mathrm{~nm}$ deep in the normal dispersion regime is believed to be the result of dispersive wave generation. The above experimental observations are supported by a comparison of the different "effective optical length scales" involved with processes potentially contributing to the supercontinuum generation. For example, the nonlinear length $L_{N L}$ is $500 \mu \mathrm{m}$ at the maximum power of $12.7 \mathrm{~W}$. In contrast, the soliton fission length [9] $L_{\text {Fission }}=\left(L_{N L} L_{D}\right)^{1 / 2}=6.3 \mathrm{~cm}$ is much larger than the length of the waveguide. Therefore, it is expected that the main processes governing the supercontinuum generation will be modulation instability and four wave mixing [2], as indeed observed in the experimental data.

In order to analyze the supercontinuum spectrum beyond the $2.5 \mu \mathrm{m}$ maximum range of our OSA, a Fourier Transform Infrared spectrometer (FTIR) equipped with a liquid-nitrogen cooled InAs detector was used. Figure 1(b) shows that the supercontinuum spectrum extends to a maximum wavelength of $2.55 \mu \mathrm{m}$. This long-wavelength limit originates from the FWM phase matching conditions, and the associated extent of the MI(2) peak. While it is possible that there are spectral components from cascaded FWM extending beyond $2.55 \mu \mathrm{m}$, any other peaks present are not visible due to the high noise floor of the InAs detector.
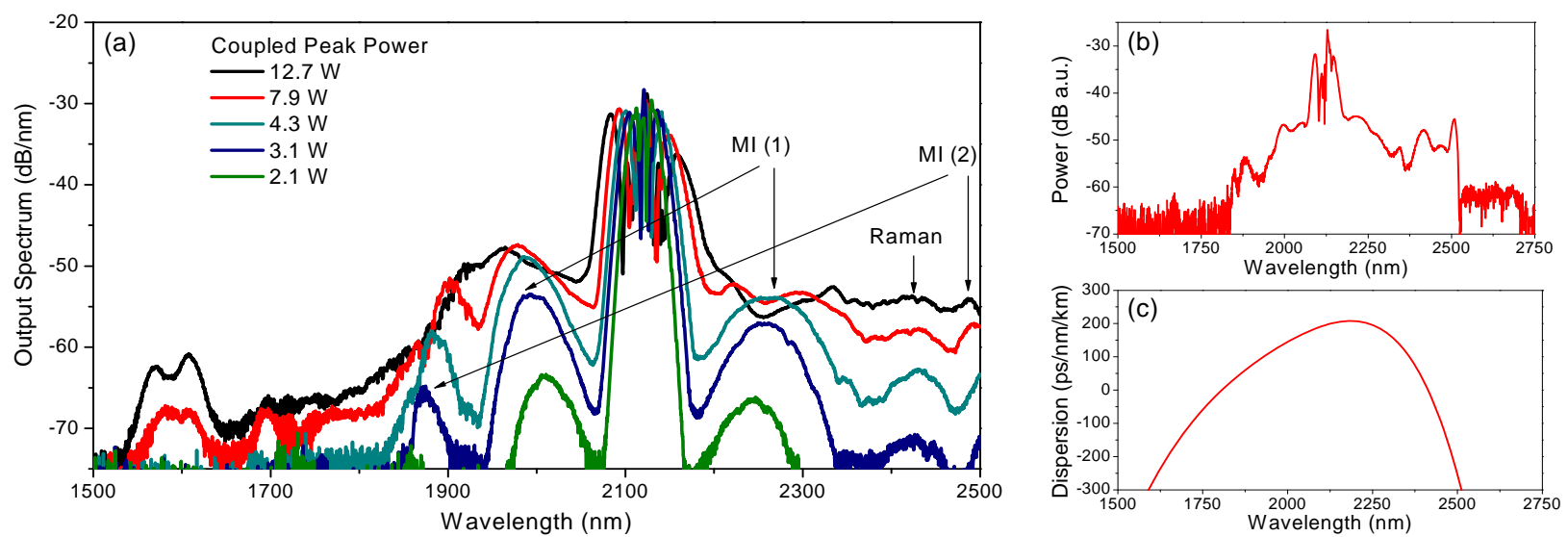

Fig. 1: (a) Power-dependent pump transmission spectra. (b) FTIR output spectrum with $P_{\text {pump }}=12.7 \mathrm{~W}$. (c) Group velocity dispersion of the wire.

\section{Conclusion}

We have demonstrated a supercontinuum generated from $2.12 \mu \mathrm{m}$ wavelength picosecond pump pulses propagating through dispersion-engineered silicon wire waveguides. Low values of anomalous dispersion at the mid-infrared pump wavelength result in a broad supercontinuum spanning the range from $1.53 \mu \mathrm{m}$ up to $2.55 \mu \mathrm{m}$.

\section{References}

[1] K. Mori et al., "Analysis and design of supercontinuum pulse generation in a single-mode optical fiber," J. Opt. Soc. Am. B 18, $1780,2001$.

[2] J. M. Dudley et al., "Supercontinuum generation in photonic crystal fiber," Rev. Mod.Phys. 78, 1135, 2006.

[3] D. Duchesne et al., "Supercontinuum generation in a high index doped silica glass spiral waveguide," Opt. Express 18, $923,2010$.

[4] M. R. Lamont et al., "Supercontinuum generation in dispersion engineered highly nonlinear $(\gamma=10 / \mathrm{W} / \mathrm{m}) \mathrm{As}_{2} \mathrm{~S}_{3}$ chalcogenide planar waveguide," Opt. Express 16, 14938, 2008.

[5] I-Wei Hsieh et al, "Supercontinuum generation in silicon photonic wires," Opt. Express 15, 15242, 2007.

[6] X. Liu et al., "Mid-infrared optical parametric amplifier using silicon nanophotonic waveguides," Nature Photonics 4, 557, 2010.

[7] S. Zlatanovic et al. "Mid-infrared wavelength conversion in silicon waveguides using ultracompact telecom-band-derived pump source," Nature Photonics 4, 561, 2010.

[8] R. K. W. Lau, et al., "Mid-infrared broadband continuous-wave parametric-mixing in silicon nanowaveguides," in OSA Frontiers in Optics, (Rochester, NY, 2010), FTuC3.

[9] C.-M. Chen et al., "Nonlinear pulse compression in optical fibers: scaling laws and numerical analysis," J. Opt. Soc. Am. B 19, $1961,2002$. 